\title{
CFD Analysis of Wind-induced Pollutant Dispersion in Built Environment
}

\author{
Okafor Chinedu VINCENT, Onyekachukwu Gozie ODENIGBO \& Okeke Arinze UCHENNA. \\ Nnamdi Azikiwe University \\ Awka, Nigeria
}

\begin{abstract}
The paper analyzed the wind-induced pollutant dispersion in a built environment using computational fluid dynamics simulation. SF6 was used as the tracer gas released from a roof stack. The steady-state analysis used to develop the adaptive mesh was carried out using the RNG-KE turbulence model. The computational domain and boundary condition for the wind flow on the urban area was adopted as recommended by the Cost guideline. Also, the SF6 species was modeled as a passive scalar transport convection-diffusion equation. From the result, it was observed that the funnel effect and vortex shedding effect reduces the concentration level of pollutants within a locality.
\end{abstract}

Key Words: CFD, Wind Power, Pollutant Dispersions.

\section{INTRODUCTION}

Determining air and contaminants in an urban environment is a very complex problem influenced by surrounding buildings [1].

Air pollution can be defined as the presence of contaminants in the air at a concentration that interferes with human health or welfare.

Recently, the use of numerical analysis involving computational fluid dynamics to predict wind around buildings and pollutant dispersion has gained more popularity in recent years [1].

The aim in this research is to analyze the wind-induced pollution dispersion in the built environment.

According to [2], accurate computational fluid dynamics simulation of this complex process requires careful simulation of each of its constituents:

1. The inaccurate atmospheric boundary layer

2. The turbulent wind flow around the buildings submerged in the atmospheric boundary layer

3. The transport process of the pollutant by convection and diffusion in the turbulent wind-flow pattern.

\section{COMPUTATIONAL DOMAIN}

A computational fluid dynamics simulation of airflow and pollutant dispersion was carried out around group of building configurations with legends: H1, H2, L1 and L2.

SF6 was used as a tracer gas and released from a stack located on the roof of the building. 


\section{International Journal of Engineering Research and Advanced Technology (IJERAT)}

DOI: $\underline{\text { 10.31695/IJERAT.2021.3603 }}$

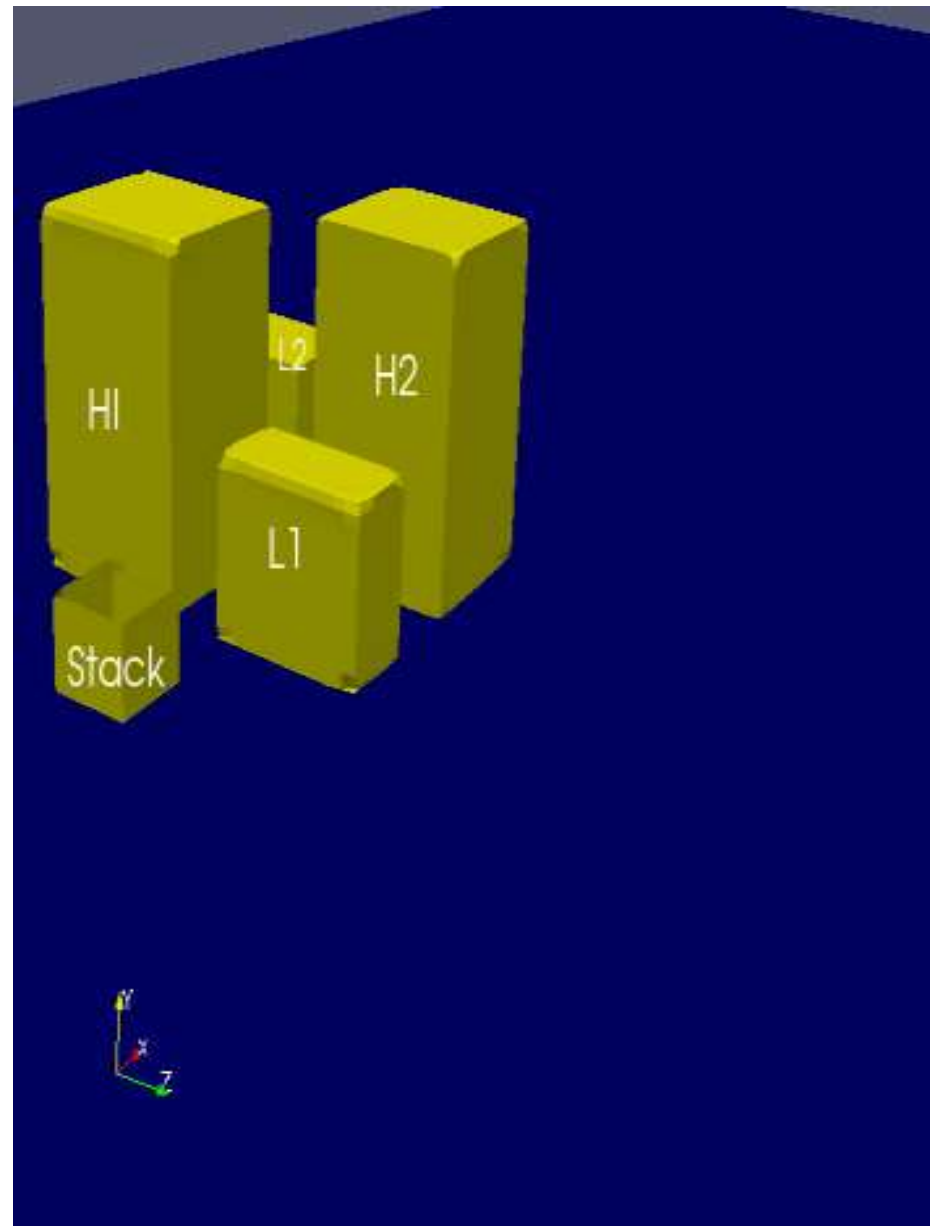

Figure 1: Array of building models

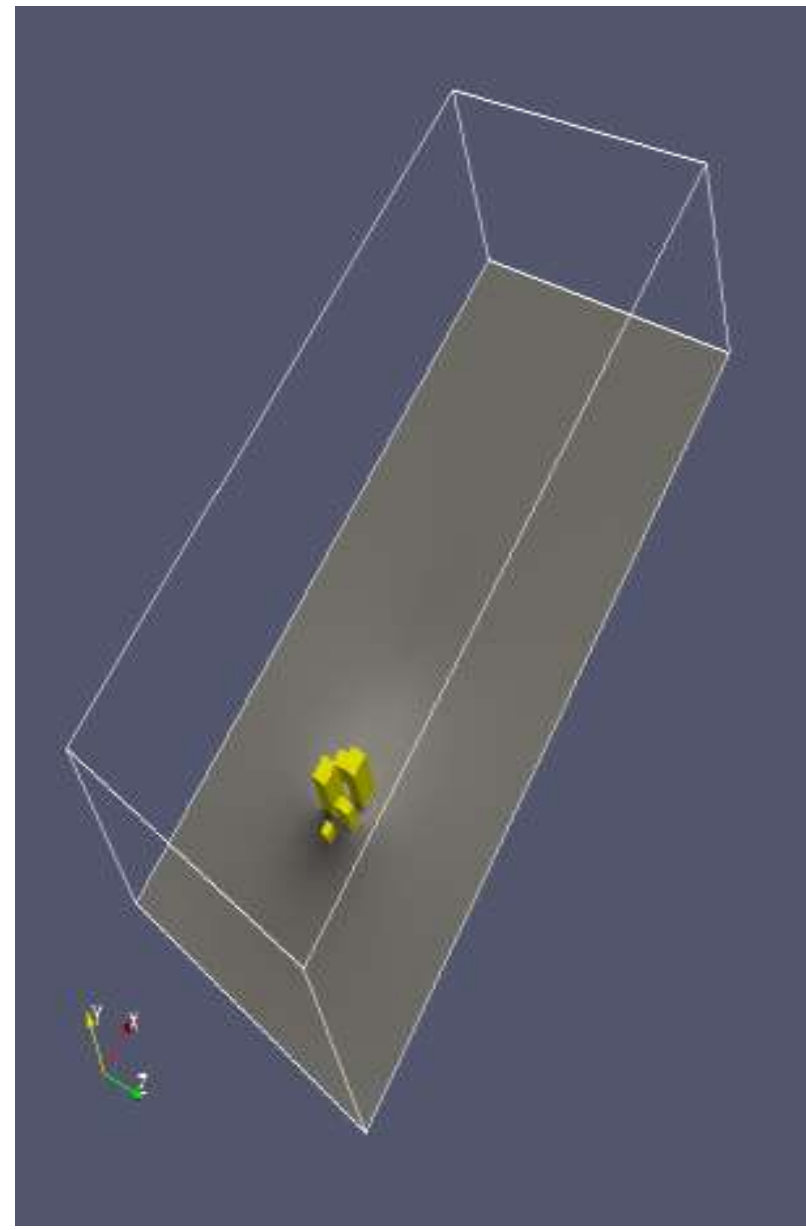

Figure 2: Computational Domain

The computational domain used for the CFD study was adopted according to recommendations by [3], the inlet, the lateral and the top boundary away from the built area is at $5 \mathrm{H}$ while the outflow boundary is $15 \mathrm{H}$, leading to a blockage ratio of $1.8 \%$. Where $\mathrm{H}$ represents the height of the building. $15 \mathrm{H}$ was recommended at the outflow boundary to develop equilibrium with the inlet conditions and also prevent backflow.

\subsection{Boundary Condition}

At the inflow boundary layer, the mean velocity profile is usually obtained from the log profile corresponding to the upwind terrain via the roughness length $Z_{0}$.

For steady RANS simulation, the mean velocity profile and turbulence quantity are obtained based on the formula suggested by [4], in which the vertical profile for wind speed $\left(u_{z}\right)$, turbulent kinetic energy $\left(k_{(z)}\right)$ and dissipation energy $(\epsilon)$ in the atmospheric boundary layer assumes a constant shear stress with height as follows:

$$
\begin{aligned}
& u_{z}=\frac{\mathrm{u}_{\mathrm{ABL}}^{*}}{\kappa} \operatorname{In}\left(\frac{Z+Z_{0}}{Z}\right) \\
& k_{(z)}=\frac{\mathrm{u}_{A B L}^{2}}{\sqrt{ } C_{\mu}}
\end{aligned}
$$


International Journal of Engineering Research And Advanced Technology, Vol.7 (4), April -2021

$$
\epsilon=\frac{\mathrm{u}_{A B L}^{* 3}}{\kappa\left(z+z_{0}\right)}
$$

Where $u_{A B L}^{*}$ is the friction velocity derived from a reference wind speed of $3.40 \mathrm{~m} / \mathrm{s}$ at a reference height $(Z)$ of $10 \mathrm{~m}$ and inlet roughness $\left(Z_{0}\right)$ of 0.1 .

Symmetry boundary condition was imposed on the lateral and top boundary following recommendation by [3].

On the ground, a rough wall was specified to model the effect of the ground roughness. According to [5],

$$
k_{S_{A B L}=20 y_{0}}
$$

Where $y_{0}$ is aerodynamic roughness length $=0.1 \mathrm{~m}$, roughness constant $\left(c_{s}\right)=0.5$.

No slip boundary type was specified for the wall velocity

The concentration of SF6 species in the air was modelled as a passive scalar $(\varphi)$ transport into the convection-diffusion equation in the form:

$$
\partial \varphi \partial t+\nabla \cdot(\cup \varphi)=\nabla \cdot(D \nabla \varphi)+S \varphi
$$

Where, $\mathrm{U}$ is velocity obtained from the momentum equation, $\mathrm{D}$ is diffussity coefficient and $S \varphi$ is the source term.

\section{SOLVER SETTING}

The commercial code SIMFLOW was used to solve the 3D steady RANS equations with the RNG-KE Turbulence model [6]. The simple algorithm was used for pressure velocity coupling. Second order discretization scheme was used for the viscous term and convective term of the governing equation.

In order to access convergence of a solution, both the scaled residue and the wind speed in some selected point of the computational domain was monitored as a function of the number of iteration. Convergence was observed when the scaled residuals reached a minimum of $10^{-6}, 10^{-4}, 10^{-4}$ for $\mathbf{x}-, \mathbf{y}$ - and $\mathbf{z}$-velocity, $10^{-5}$ for $\boldsymbol{k}$ and $10^{-6}$ for $\boldsymbol{\varepsilon}$ respectively.

\section{RESULT AND DISCUSSION}

From the CFD result in Fig 5, the pollutant concentration evolution initially follows the flow streamline around the release region. As the pollutant exits the stack, the pollutant rapidly mixes with the wind resulting in a lesser level of pollutant concentration beyond the stack region. 
International Journal of Engineering Research And Advanced Technology, Vol.7 (4), April -2021

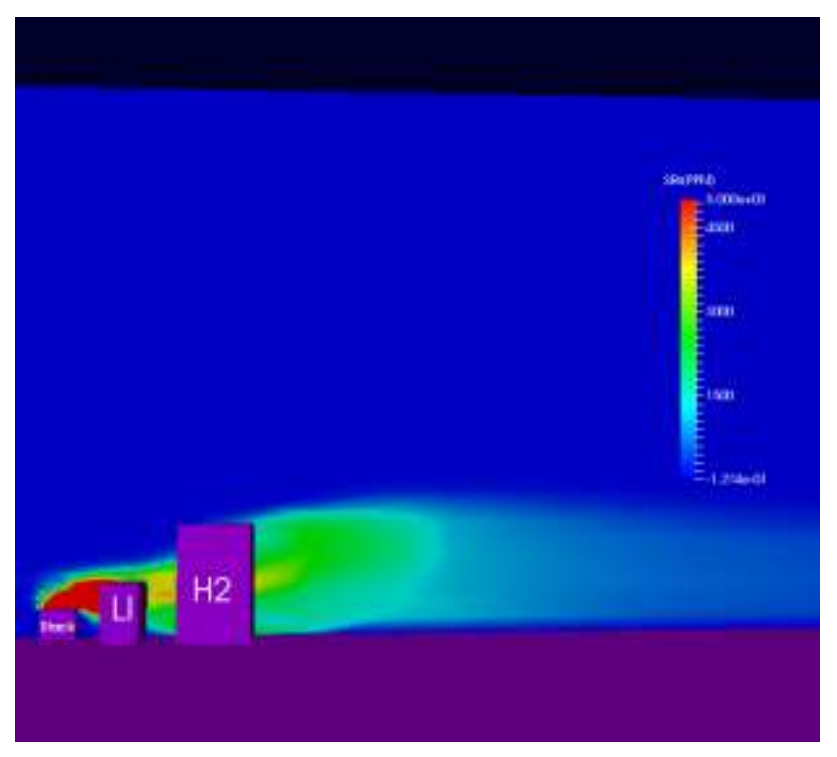

Figure 3: Slice view of Pollutant Dispersion

More so, the flow of the wind through the small spaces between adjacent buildings further increases the wind speed thereby lowering the level of pollutant concentration due to the funneling effect. Wind funnel effect is a phenomenon whereby wind will tend to increase in wind speed as they squeeze through two adjacent surfaces. Because of the funneling effect, the wind tends to disperse in all direction and the pollutant also follows this by covering a larger area of the computational domain but at a reduced pollutant concentration level.

Lower level of concentration of the pollutant was also observed at the right side of the computational domain. These lower concentrations displayed a periodic movement which was caused as a result of the periodic vortex shedding noticed at the wake of building $\mathrm{H} 1$ and $\mathrm{H} 2$. Though the pollutant concentrations at the region of the stack source seem relatively static, the pollutant concentration in the flow downstream tends to be remarkably unsteady. 
International Journal of Engineering Research and

Advanced Technology (IJERAT)

DOI: $10.31695 /$ IJERAT.2021.3603
E-ISSN : 2454-6135

Volume.7, Issue 4

April -2021

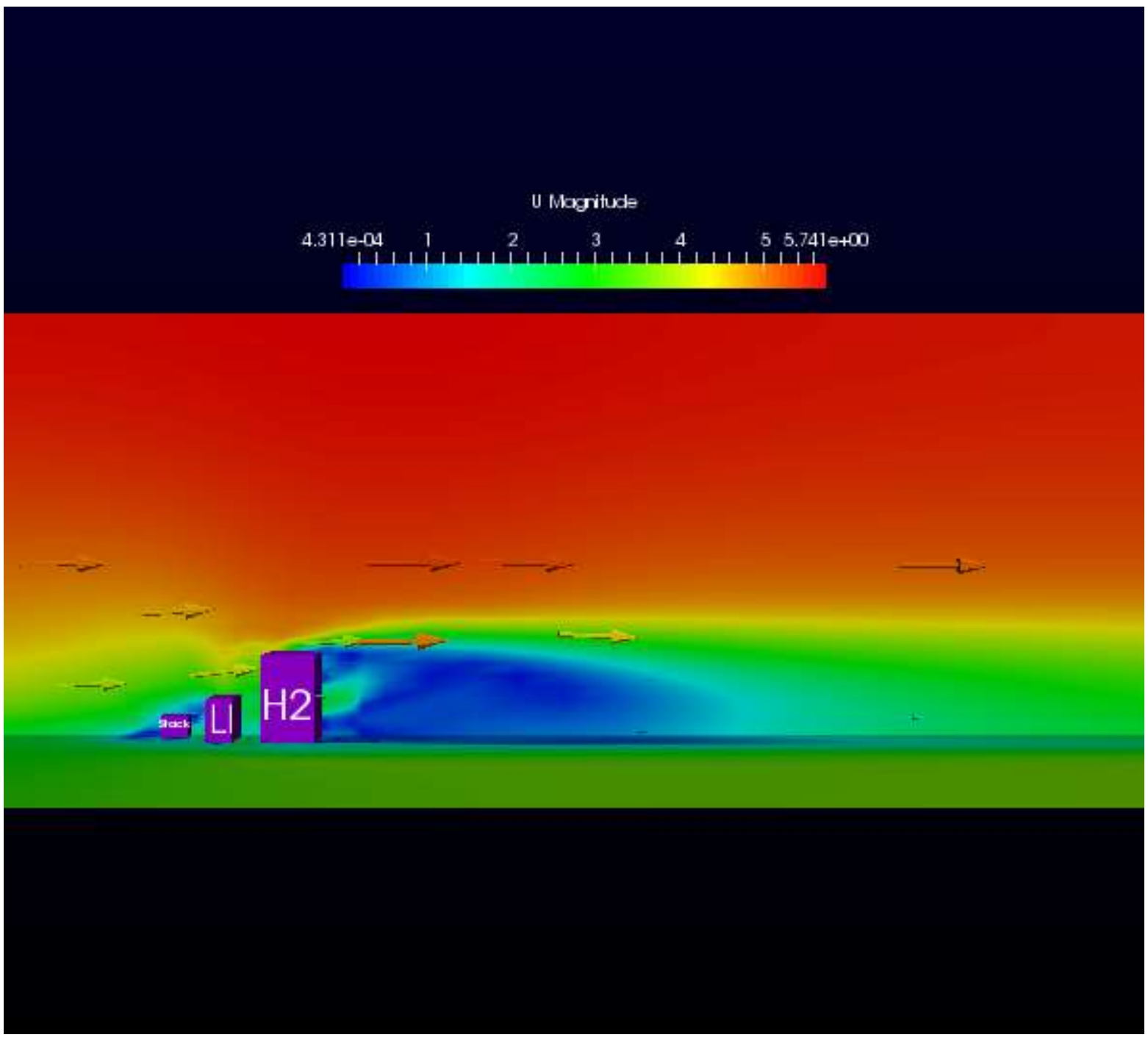

Figure 4: Side view of Velocity vector and magnitude of velocity field 
International Journal of Engineering Research And Advanced Technology, Vol.7 (4), April -2021

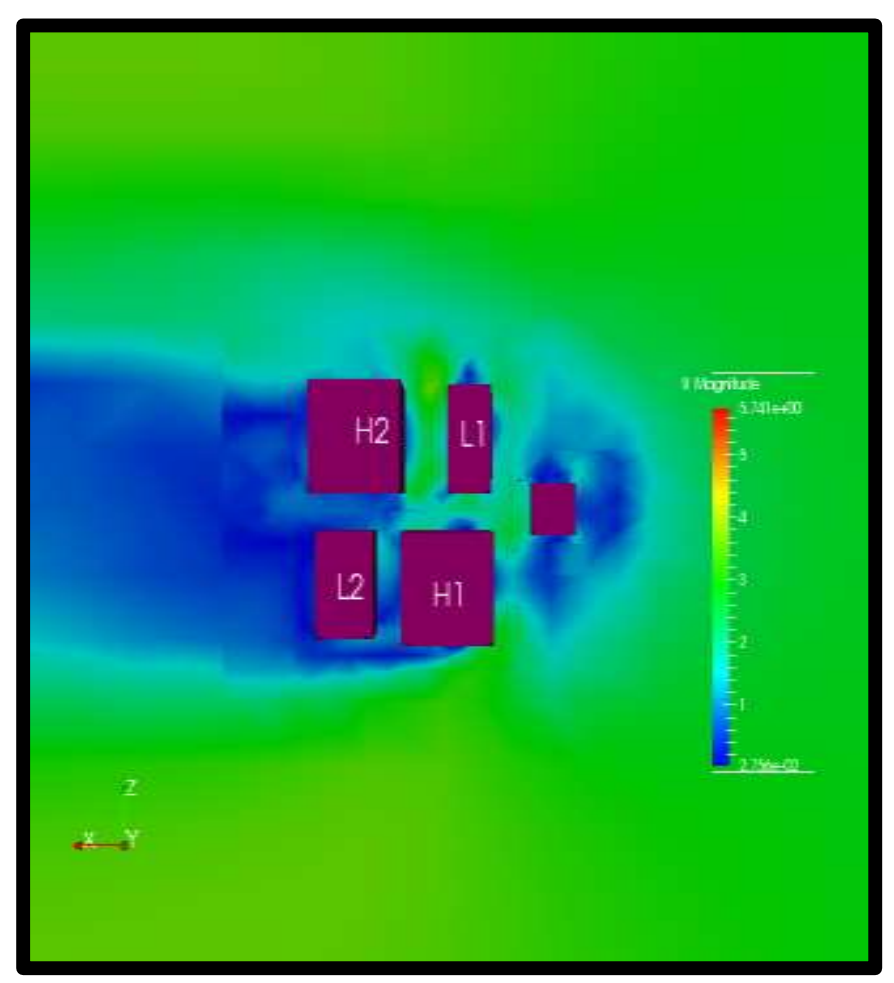

Figure 5: Top View of velocity magnitude 
International Journal of Engineering Research And Advanced Technology, Vol.7 (4), April -2021

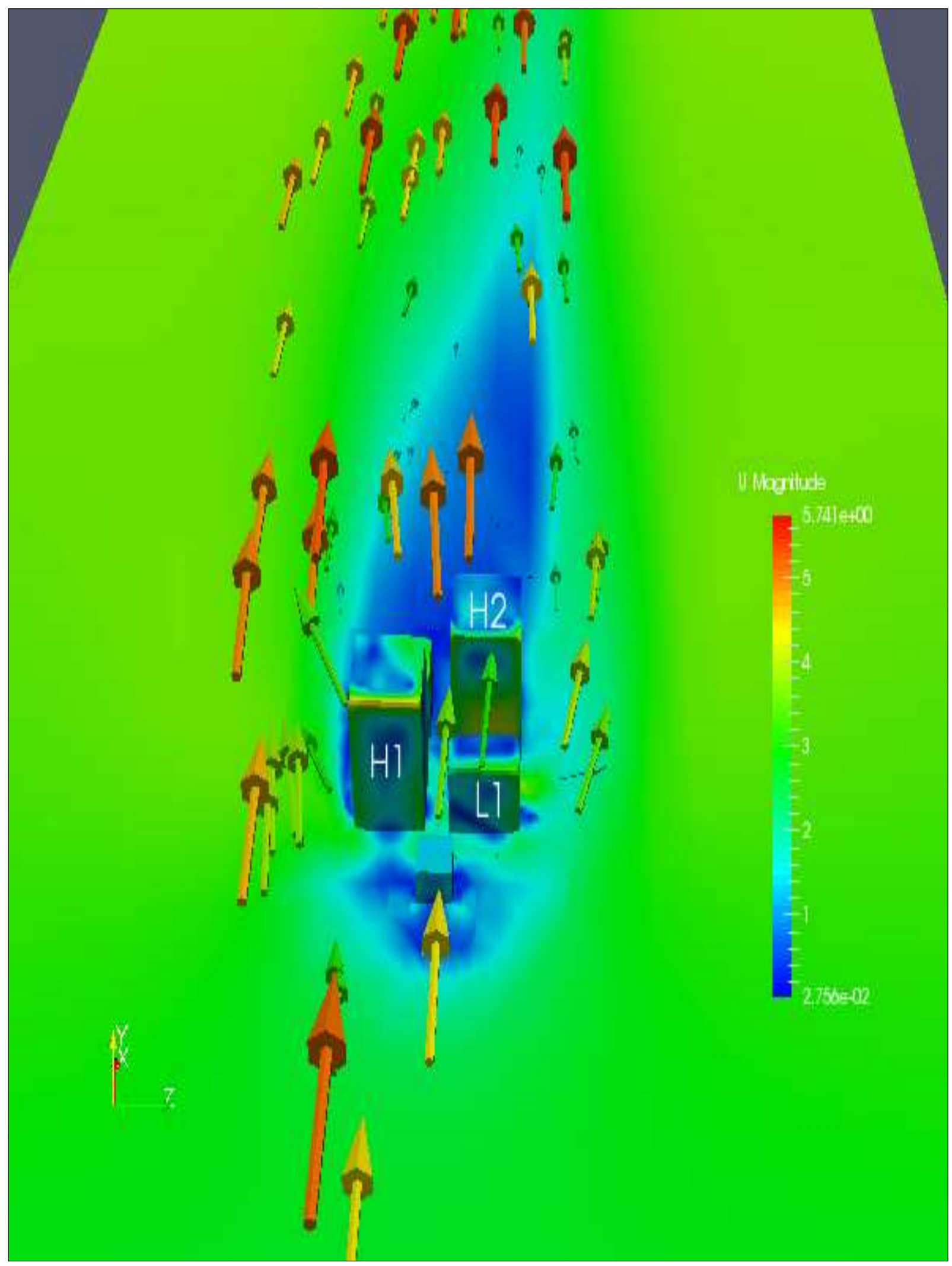

Fig 6: Top view of velocity vector and magnitude of velocity field 

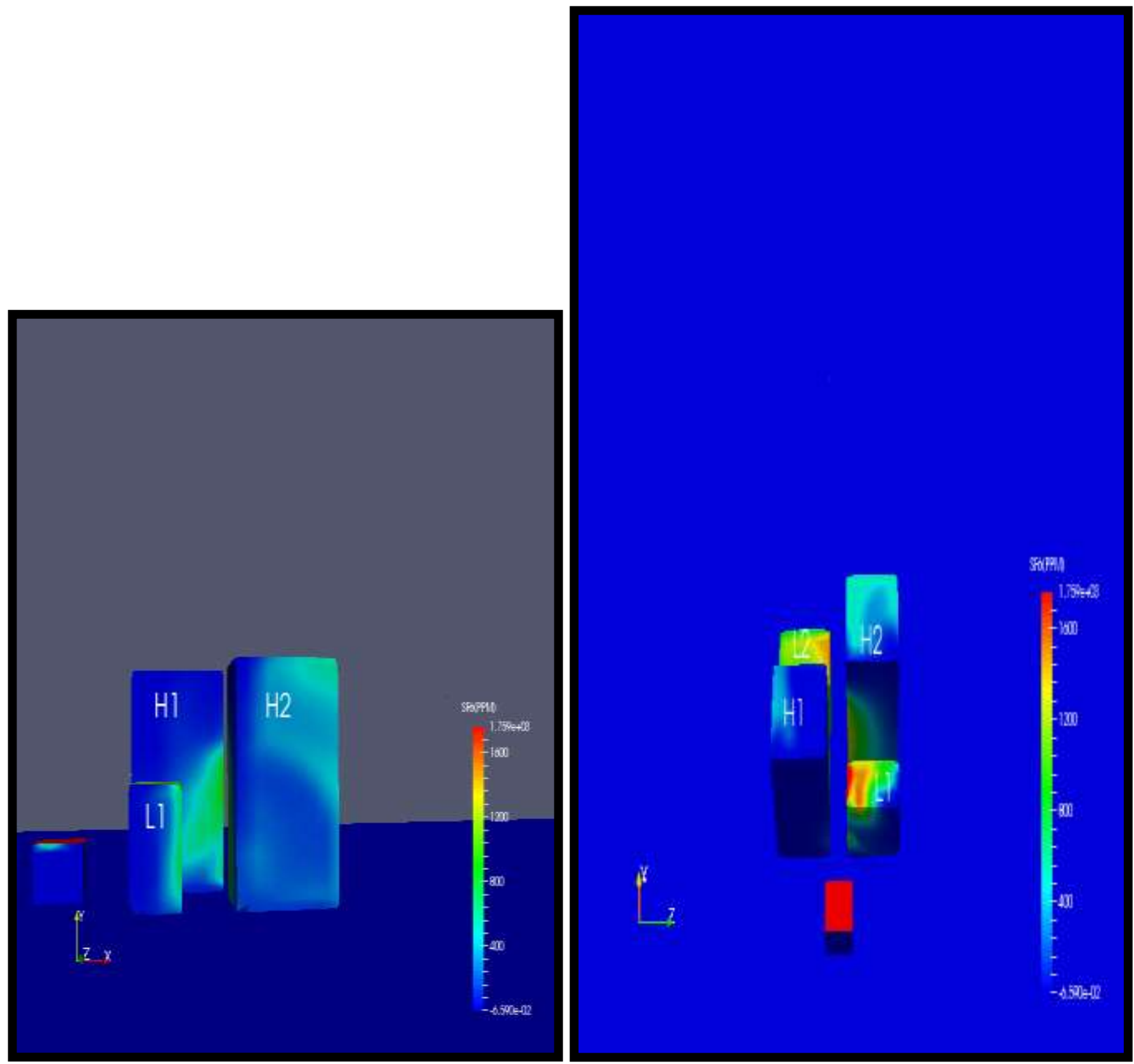

Fig 7: Top view and Right view of Pollutant Concentration around the Built Environment

\section{CONCLUSION}

The purpose of this research was to analyze the wind induced pollutant dispersion in a built environment using computational fluid dynamics simulation.

Based on the analysis completed, it can be concluded that funneling effect between adjacent buildings and the vortex shedding effects experienced at the wake of rectangular building structures respectively has the potential to enhance the dispersion of gaseous pollutants thereby reducing the pollutant concentration in an urban built environment. 


\section{REFERENCES}

[1]. N.Al-khalidy, "City scale pollutant dispersion modelling utilising a combination of computational fluid dynamics and standard air-quality simulation”. International journal of mechanics. Vol. 11, No.2, pp.210-217,2017

[2]. P.Gousseau, B.Blocken, T.Staphopoulos and G.J.F. Van Heighst. "CFD simulation of pollutant dispersion around isolated buildings on the role of connective and turbulent mass fluxes in the prediction accuracy". Journal of Hazardous material Vol.194,PP.422-434,2011

[3]. J.Franke, C.Hirsch, A.Jensen, H.Krus, M.Schatzmann, P.Westbury, S.Miles, J.Wisse and N.Wright. "Recommendations on the use of CFD in wind engineering", COST ActionC14: Impact of Wind and storm on city life and Built Environment, von Karman Institute for Fluid Dynamics,2004.

[4]. P.Richards and R.Hosey. "Appropriate boundary conditions for computational wind engineering model using the K-e model". J.wind eng.ind.aerod. Vol.46, No.47, PP.145-153, 1993.

[5]. B.Blocken, T.Stathopoulos, J.Carmeliet, CFD simulation of the atmospheric boundary layer: wall function problems.atmos.enviro.41 (2), 228-252.

[6]. V.Yakhot, S.Orszag, S.Thangam, T.Gatski and C.Speziale. "Development of turbulence model for shear flows by a double expansion technique". physics of fluids.Vol.4,No.7,pp.1510-1520,1992. 